\title{
Synthesis of (2S, 3R)-2-allyl-3-furyl cyclopentanone. An enantioselective strategy towards the synthesis of phorbol
}

\author{
Vanida Chairgulprasert, Michael G. B. Drew, Archie Jahans, and Laurence M. Harwood \\ Department of Chemistry, University of Reading, Whiteknights, Reading RG6 6AD, UK. \\ E-mail: l.m.harwood@reading.ac.uk
}

\section{Dedicated to Professor Jimmy Bull on the occasion of his retirement}

\begin{abstract}
(1S, 5R)-5-(2-furyl)-2-oxocyclopentanecarboxylate 10 was synthesised by asymmetric conjugate addition of lithium difurylcyanocuprate to the chiral substrate $\mathbf{9}$ in high diastereoisomeric excess (>95 \%). After transesterification, allylation and subsequent decarbomethoxylation, (2S, 3R)-2allyl-3-furyl cyclopentanone $\mathbf{1 3}$ was obtained in $>86 \%$ ee.
\end{abstract}

Keywords: Chiral, cyclopentenone, phorbol

\section{Introduction}

Phorbol 1 and its derivatives are toxic diterpenes found in the sap of plants of the family Euphorbiaceae. ${ }^{1}$ The extracts and sap of these plants have been used in folkloric medicine as purgatives but their toxicological properties are very harsh ${ }^{2}$ and tetradecanoyl phorbol acetate has been found to be a potent tumour promoter. ${ }^{3}$ Wender has succeeded in total synthesis of phorbol, ${ }^{4}$ while other routes have yet to be completed. ${ }^{5}$ Thus far only Wender ${ }^{6 a}$ and Shibasaki ${ }^{6 b}$ have reported enantioselective strategies.

To date, we have made substantial progress in a synthetic approach to phorbol utilising the diastereocontrolled ultra-high pressure promoted intramolecular Diels-Alder reaction of furan $(\mathrm{IMDAF})^{7}$ to provide tricyclic adducts 2 with relative stereochemistry at 6 stereocentres and disposed functionality rendering them amenable to access phorbol.

The relative stereocontrol in the cycloaddition is a consequence of the trans-2, 3 relative configuration at the cyclopentanone and endo cycloaddtion of the Z-dienophile in the precursor $\mathbf{4}$ under high pressure conditions and subsequent regiocontrolled epimerisation of the cycloadducts 3 at standard pressure to furnish the desired relative stereochemistry (Figure 1). Thus, stereochemical information in the trans-2-substituted-3-furyl cyclopentanone precursor $\mathbf{4}$ is relayed and amplified during the sequence and, therefore, development of an enantiocontrolled 
approach to substrates of general structure $\mathbf{5}$ could provide precursors suitable for elaboration to the natural antipode of phorbol and its analogues.

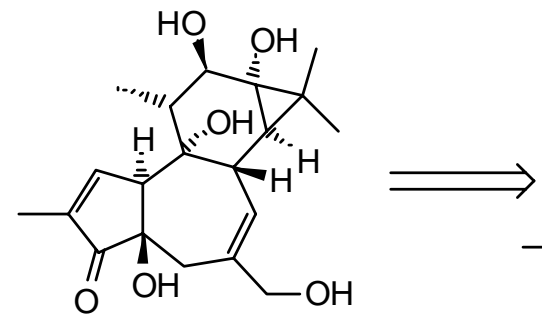

1

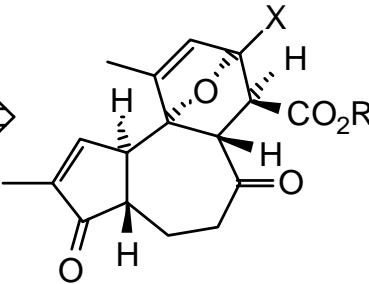

2

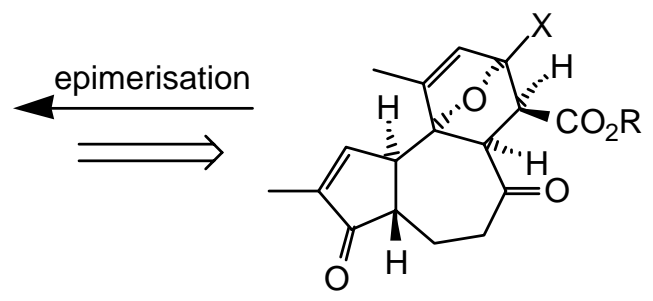

endo IMDAF $\uparrow_{\Downarrow}^{3}$

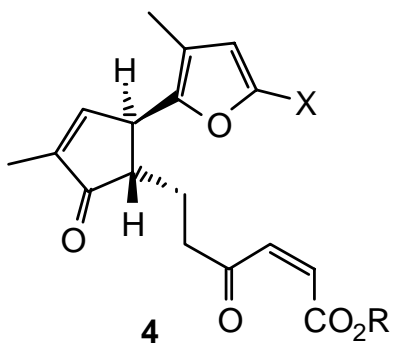

Figure 1

\section{Results and Discussion}

This paper reports a study into an enantioselective synthetic approach to (2S, 3R) 2-allyl-3-(2furyl)cyclopentanone, a central precursor to many of our studies. The key step of the synthetic approach is asymmetric conjugate addition to a chiral cyclopent-2-enone substrate. After surveying a range of chiral alcohols, transesterification of methyl (2-oxocyclopentane) carboxylate with (1R)-isoborneol sulfonamide derivative $\mathbf{6}$, an efficient chiral auxiliary for asymmetric conjugate addition to cyclopentenone substrates, ${ }^{8}$ using vanadyl (IV) acetate as catalyst ${ }^{9}$ furnished ketoester 7 in high yield (94\%). ${ }^{13} \mathrm{C}$ NMR spectroscopic analysis of 7 showed two diastereoismers in an approximate ratio of $1: 2$. Phenylselenenylation ${ }^{10}$ of both diastereomers afforded a mixture of diastereomeric selenides 8 (89:11) and oxidative deselenenylation was accomplished with MCPBA to furnish enantiomerically pure olefinic ketoester $\mathbf{9}$ as colourless needles in $86 \%$ yield after recrystallisation (Scheme 1).

At $-78^{\circ} \mathrm{C}$, lithium difuranyl cyanocuprate ${ }^{11}$ underwent diastereocontrolled conjugate addition to the least hindered $\pi$-face of 9 resulting in (1S, 5R)-5-(2-furyl)-2-oxocyclopentanecarboxylate 10 as a single diastereoisomer (>95 \% de), as shown by ${ }^{1} \mathrm{H}$ NMR and ${ }^{13} \mathrm{C}$ NMR spectroscopy. 
The 1H NMR spectrum showed keto and enol tautomers in a ratio of $4: 1$ but the alternative diastereisomer could not be detected. X-ray crystallographic analysis confirmed that the 5-(2furyl) substituent and the ester group were trans to each other and that the adduct had the required absolute stereochemistry at $\mathrm{C}-1$ and $\mathrm{C}-5$ to access the natural antipode of phorbol (Figure 2). ${ }^{12}$

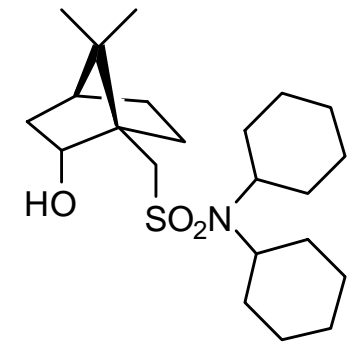

6

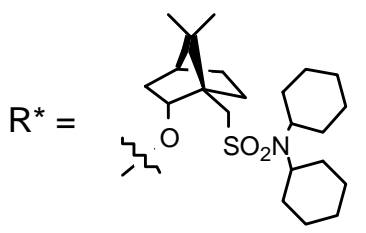

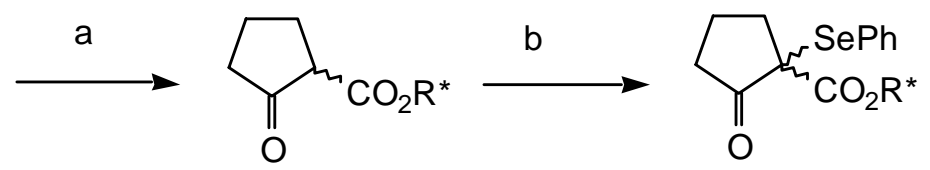

7

8

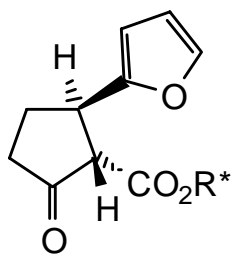

10

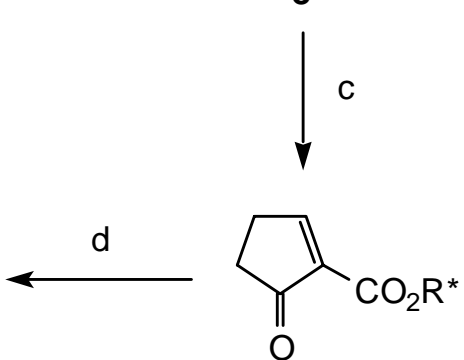

9

Scheme 1. a Methyl (2-oxocyclopentane)carboxylate, $\mathrm{V}(\mathrm{OAc})_{4}$, toluene, reflux; b. Pyridine, DCM , rt;. c. MCPBA, DCM; d. $\left(\mathrm{C}_{4} \mathrm{H}_{3} \mathrm{O}\right)_{2} \mathrm{CuCNLi}_{2}, \mathrm{BF}_{3} \cdot \mathrm{OEt}_{2},-78^{\circ} \mathrm{C}$.

Adduct 10 was efficiently transesterified using methanol in a sealed-tube at $100{ }^{\circ} \mathrm{C}$, following Ikegami's method ${ }^{13}$ to furnish the corresponding $(1 S, 5 R)$ methyl ester $\mathbf{1 1}$ cleanly in good yield (91\%) without epimerisation at C5 (Scheme 2) as confirmed by X-ray crystallographic analysis (Figure 2) ${ }^{12}$ In addition, this procedure allowed recovery of the chiral auxiliary in $85 \%$ yield with $>95 \%$ ee. Subsequently, $\mathbf{1 1}$ was allylated following the procedure of Urban ${ }^{14}$ to produce (1R, $5 R$ ) methyl-1-allyl-5-(2-furyl)-2-oxocyclopentanecarboxylate $\mathbf{1 2}$ as a single diastereoisomer in high yield (89\%), X-ray crystallographic analysis showing that the allyl group was trans to the furyl group (Figure 2).12 


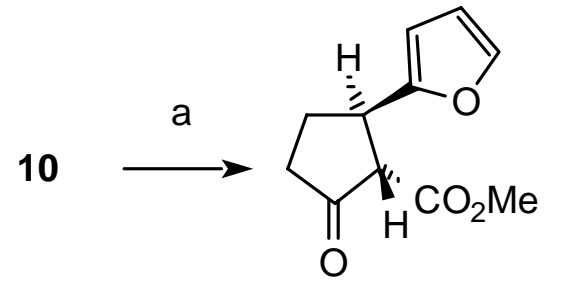

11

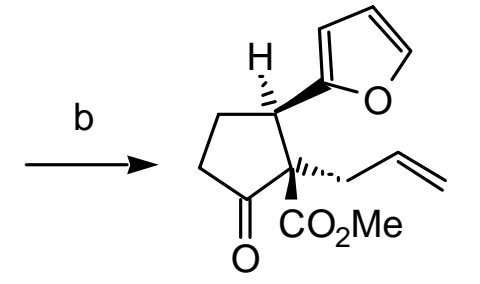

12

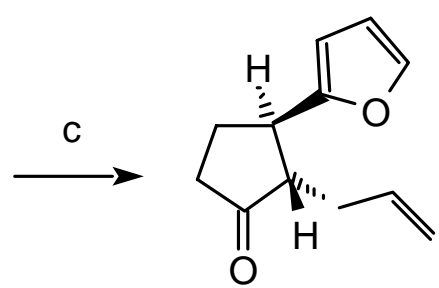

13

Scheme 2. $\mathrm{MeOH}, 100^{\circ} \mathrm{C}, 18$ h. b. i. $\mathrm{KN}\left[\mathrm{Si}(\mathrm{Me})_{3}\right]_{2}$, THF, $-78^{\circ} \mathrm{C}$, ii. $\mathrm{CH}_{2} \mathrm{CH}=\mathrm{CH}_{2} \mathrm{Br},-78^{\circ} \mathrm{C} \rightarrow$ rt. c. LiCl, HMPA, $80^{\circ} \mathrm{C}$.

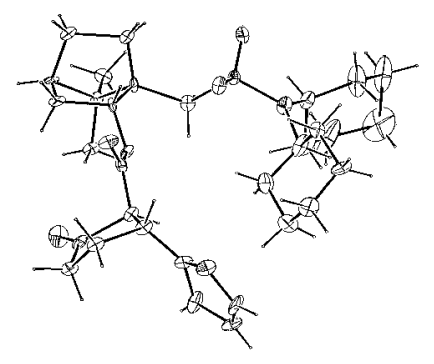

10

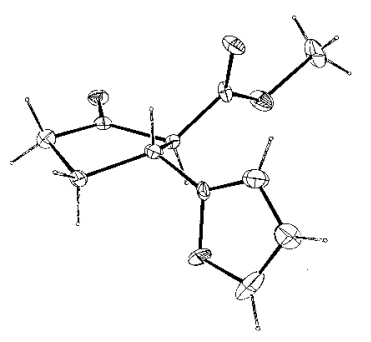

11

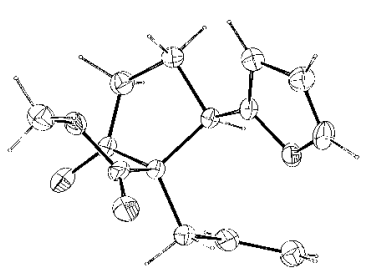

12

Figure 2. X-ray structures of adduct 10;. methyl ester 11; allylated ester 12.

Finally, the carbomethoxy group of $\mathbf{1 2}$ was removed by lithium chloride in $\mathrm{HMPA}^{15}$ affording 2allyl-3-(2-furyl) cyclopentanone 13 as a trans : cis mixture in a ratio of $7: 1$. The pure (2S, 3R)trans-2-allyl-3-(2-furyl)cyclopentanone 13 was obtained by column chromatography in $73 \%$ yield and possessed an enantiomeric excess greater than $86 \%$ as determined using Eu(tfac) 3 as a chiral shift reagent and comparison with racemic trans-2-allyl-3-furyl cyclopentanone; the resonances at $\delta 5.08-5.00 \mathrm{ppm}$ corresponding to the two terminal vinyl protons being clearly split in the ${ }^{1} \mathrm{H}$ NMR spectrum,.

In conclusion, an experimentally straightforward enantioselective synthesis of (2S, 3R)-2allyl-3-(2- furyl)cyclopentanone (13) has been accomplished. This methodology will be applied to our enantioselective synthetic approach to phorbol.

\section{Experimental Section}

General Procedures. Preparation of chiral ester 7. A mixture of (1R)-5 (3 g, $7.54 \mathrm{mmol}$ ), methyl-2-oxocyclopentane 6 (1.06 mL, $8.54 \mathrm{mmol})$ and vanadyl (IV) acetate (212.5 mg, $1.16 \mathrm{mmol})$ in toluene $(80 \mathrm{~mL})$ was refluxed for $18 \mathrm{~h}$. The reaction mixture was then filtered through Celite ${ }^{\circledR}$ and the filtrate was concentrated in vacuo. The crude solid was recrystallised 
(DCM : light petroleum ether) to give the desired product as a colourless needles and the mother liquors were further purified by flash column chromatography (hexane : EtOAc, 1:1) to give an additional quantity of the desired product ( $3.6 \mathrm{~g}, 94 \%$ combined yield) $\mathrm{mp} 181-182^{\circ} \mathrm{C}$; $v_{\max }$ $\left(\mathrm{CHCl}_{3}\right) 1754,1728,1456,1393,1144,1050 \mathrm{~cm}^{-1} ; \delta_{\mathrm{H}}\left(250 \mathrm{MHz}, \mathrm{CDCl}_{3}\right): 4.97-4.94(1 \mathrm{H}, \mathrm{m}$, CHOCO), $3.25\left(1 \mathrm{H}, \mathrm{d}, \mathrm{J}=13.3 \mathrm{~Hz}, \mathrm{HCHSO}_{2}\right), 3.28-3.09\left(2 \mathrm{H}, \mathrm{m}, 2 \mathrm{CHNSO}_{2}\right), 3.05(1 \mathrm{H}, \mathrm{t}, \mathrm{J}=$ $\left.8.8 \mathrm{~Hz}, \mathrm{CO}_{2} \mathrm{CHCO}\right), 2.66\left(1 \mathrm{H}, \mathrm{d}, \mathrm{J}=13.3 \mathrm{~Hz}, \mathrm{HCHSO}_{2}\right), 2.47-2.07,2.07-1.59$ and 1.28-0.96 (33H, m, $\left.\left(\mathrm{CH}_{2}\right)_{3} \mathrm{CO},\left(\mathrm{CH}_{2}\right)_{2} \mathrm{CHCH}_{2}, 2\left(\mathrm{CH}_{2}\right)_{5} \mathrm{CHN}\right), 1.05$ (3H, s, $\left.\mathrm{CH}_{3}\right), 0.86\left(3 \mathrm{H}, \mathrm{s}, \mathrm{CH}_{3}\right)$ ), $\delta_{\mathrm{C}}$ NMR (250 MHz, $\mathrm{CDCl}_{3}$ ): 212.4, 167.7, 79.3, 57.4, 54.4, 53.8, 49.4, 49.1, 44.4, 39.4, 38.1, 32.8, 32.7, 27.0, 26.5, 26.4, 26.2, 25.2, 20.4, 19.9 diastereoisomer 211.6, 167.1, 78.8, 57.2, 54.8, 49.6, 38.0, 33.3, 32.1, 30.1, 27.1, 26.3, 20.9; HRMS Found 508.3108, $\mathrm{C}_{28} \mathrm{H}_{46} \mathrm{NSO}_{5}{ }^{+}\left(\mathrm{MH}^{+}\right)$requires 508.3096.

Preparation of selenides 8. Dry pyridine $(0.31 \mathrm{~mL}, 3.9 \mathrm{mmol})$ and a solution of ester 7(1.98 g, $3.9 \mathrm{mmol})$ in DCM $(25 \mathrm{~mL}$ ) were added sequentially to a solution of phenylyselenenyl bromide $(0.94 \mathrm{~g}, 3.9 \mathrm{mmol})$ in $\mathrm{DCM}(25 \mathrm{~mL})$ at $0^{\circ} \mathrm{C}$. The reaction was warmed to room temperature and stirred for $12 \mathrm{~h}$. The resulting mixture was washed with $1 \mathrm{M} \mathrm{HCl}$, dried $\left(\mathrm{MgSO}_{4}\right)$ and concentrated in vacuo. The two isomeric products were obtained after purification by flash column chromatography (3:1, hexane: EtOAc) as a colourless solid $(2.27 \mathrm{~g}, 87 \%) \mathrm{mp} 144^{\circ} \mathrm{C}$; $v_{\max }\left(\mathrm{CHCl}_{3}\right) 1733,1721,1325,1143,1049 \mathrm{~cm}^{-1} ; \delta_{\mathrm{H}}\left(250 \mathrm{MHz}, \mathrm{CDCl}_{3}\right): 7.58-7.54(2 \mathrm{H}, \mathrm{dd}$, $\mathrm{J}=1.3,8.0 \mathrm{~Hz}, P h), 7.37-7.26$ (3H, m, $P h), 4.96(1 \mathrm{H}, \mathrm{dd}, \mathrm{J}=2.1,6.9 \mathrm{~Hz}, \mathrm{CHOCO}), 3.37(1 \mathrm{H}, \mathrm{d}, \mathrm{J}=$ $\left.13.3 \mathrm{~Hz}, \mathrm{HCHSO}_{2}\right), 3.24-3.16\left(2 \mathrm{H}, \mathrm{m}, 2(\mathrm{CH}) \mathrm{NSO}_{2}\right), 2.53\left(1 \mathrm{H}, \mathrm{d}, \mathrm{J}=13.3 \mathrm{~Hz}, \mathrm{HCHSO}_{2}\right), 2.38$ (2H, t, J= 7.5 Hz, CH $\mathrm{CH}_{2} \mathrm{CO}$ ), 1.95-1.05 (31H, m, $\mathrm{CH}_{2} \mathrm{CH}_{2} \mathrm{C},\left(\mathrm{CH}_{22} \mathrm{CHCH}_{2} \mathrm{CHO}, 2(\mathrm{CH} 2)_{5} \mathrm{CHN}\right)$, $1.06\left(3 \mathrm{H}, \mathrm{s}, \mathrm{CH}_{3}\right), 0.85\left(3 \mathrm{H}, \mathrm{s}, \mathrm{CH}_{3}\right)$ diastereoisomer : $7.50(2 \mathrm{H}, \mathrm{d}, \mathrm{J}=7.5 \mathrm{~Hz}, \mathrm{Ph}), 4.94(1 \mathrm{H}, \mathrm{dd}$, $\mathrm{J}=2.8,7.8 \mathrm{~Hz}, \mathrm{CHOCO}), 3.41\left(1 \mathrm{H}, \mathrm{d}, \mathrm{J}=13.3 \mathrm{~Hz}, \mathrm{HCHSO}_{2}\right), 2.19$ (2H, t, J= 7.5 Hz, CH $\mathrm{CO}_{2}$, 1.03, (3H, s, $\left.\mathrm{CH}_{3}\right), 0.98$ (3H, s, $\left.\mathrm{CH}_{3}\right)$; $\delta_{\mathrm{C}} \mathrm{NMR}\left(250 \mathrm{MHz}, \mathrm{CDCl}_{3}\right)$ : 208.5, 168.3, 137.7, 131.8, 130.0, 129.5, 129.4, 128.1, 126.9, 79.5, 58.0, 57.5, 54.3, 50.2, 49.7, 44.7, 39.2, 38.0, 33.9, 32.3, 31.3, 27.5, 26.9, 26.6, 25.6, 20.8, 20.4, 19.6; HRMS Found 663.2489, $\mathrm{C}_{34} \mathrm{H}_{50} \mathrm{NSO}_{5} \mathrm{Se}^{+}\left(\mathrm{MH}^{+}\right)$ requires 663.2496.

Preparation of chiral cyclopentenone 9. A solution of MCPBA (55 \%, $332 \mathrm{mg}, 1.06 \mathrm{mmol}$ ) in DCM $(10 \mathrm{~mL})$ was added to a solution of selenide $8(554.7 \mathrm{mg}, 0.8 \mathrm{mmol})$ in DCM $(10 \mathrm{~mL})$ at room temperature and the reaction was then stirred for $3 \mathrm{~h}$. The resulting mixture was washed with saturated $\mathrm{NaHCO}_{3}, \mathrm{NaHSO}_{3}$, dried $\left(\mathrm{MgSO}_{4}\right)$ and concentrated in vacuo. The crude solid was washed with ether $(3 \times 10 \mathrm{~mL})$ and recrystallised (DCM: light petroleum ether) to give colourless needles ( $364 \mathrm{mg}, 86 \%) \mathrm{mp} 165-166^{\circ} \mathrm{C} ;[\alpha]_{\mathrm{D}}+38.8$ (c 1.02, $\left.\mathrm{CHCl}_{3}\right) ; v_{\max }\left(\mathrm{CHCl}_{3}\right)$ 1748, 1716, 1322, 1290, 1143, $1108 \mathrm{~cm}^{-1} ; \delta_{\mathrm{H}}\left(250 \mathrm{MHz}, \mathrm{CDCl}_{3}\right): \delta 8.49(1 \mathrm{H}, \mathrm{t}, \mathrm{J}=2.8 \mathrm{~Hz}$, $\mathrm{CH}=\mathrm{C}), 5.11(1 \mathrm{H}, \mathrm{dd}, \mathrm{J}=2.5,7.5 \mathrm{~Hz}, \mathrm{CHOCO}), 3.46\left(1 \mathrm{H}, \mathrm{d}, \mathrm{J}=13.3 \mathrm{~Hz}, \mathrm{HCHSO}_{2}\right), 3.17-3.11$ $\left.(2 \mathrm{H}, \mathrm{m}, 2 \mathrm{CHNSO})_{2}\right), 2.70-2.68\left(2 \mathrm{H}, \mathrm{m}, \mathrm{CH}_{2} \mathrm{CH}=\mathrm{CCO}\right), 2.63\left(1 \mathrm{H}, \mathrm{d}, \mathrm{J}=13.3 \mathrm{~Hz}, \mathrm{HCHSO}_{2}\right)$, 2.52-2.50(2H, m, $\left.\mathrm{CH}_{2} \mathrm{CO}\right), 1.79-1.57$ and 1.53-0.88 (27H, m, $\left.\left(\mathrm{CH}_{2}\right)_{2} \mathrm{CHCH}_{2}, 2\left(\mathrm{CH}_{2}\right)_{5} \mathrm{CHN}\right), 1.06$ (3H, s, $\left.\mathrm{CH}_{3}\right), 0.88\left(3 \mathrm{H}, \mathrm{s}, \mathrm{CH}_{3}\right)$ ), $\delta_{\mathrm{C}}\left(250 \mathrm{MHz}, \mathrm{CDCl}_{3}\right): 202.1,173.1,160.6,137.9,78.6,57.2$, 
53.5, 49.6, 49.1, 44.5, 33.3, 36.0, 33.0, 32.4, 30.0, 27.0, 26.5, 26.3, 25.2, 20.4, 20.0; HRMS $.2931, \mathrm{C}_{28} \mathrm{H}_{44} \mathrm{NSO}_{5}{ }^{+}\left(\mathrm{MH}^{+}\right)$requires 506.2940.

Preparation of conjugate addition adduct 10. $n$-Butyllithium $(2.5 \mathrm{M}$ in hexane, $9.14 \mathrm{~mL}$, $22.9 \mathrm{mmol})$ was added dropwise to a solution of furan $(1.73 \mathrm{~mL}, 23 \mathrm{mmol})$ in dry THF $(20 \mathrm{~mL})$ at $-40^{\circ} \mathrm{C}$ under nitrogen. The solution was slowly warmed to $0^{\circ} \mathrm{C}$ and stirred for $1 \mathrm{~h}$ then cooled to $-40^{\circ} \mathrm{C}$. The resulting furyl lithium was transferred via canula to a solution of copper cyanide $(1.04 \mathrm{~g}, 11.8 \mathrm{mmol})$ in dry THF $(100 \mathrm{~mL})$ at $-40^{\circ} \mathrm{C}$, then the mixture was slowly warmed to $0^{\circ} \mathrm{C}$. The resulting clear solution was cooled to $-78^{\circ} \mathrm{C}$, then a solution of boron trifluoride etherate $(1.4 \mathrm{~mL}, 11.6 \mathrm{mmol})$ and a solution of chiral substrate $9(577 \mathrm{mg}, 1.12 \mathrm{mmol})$ in dry THF $(20 \mathrm{ml})$ were added sequentially. The reaction was stirred at $-78^{\circ} \mathrm{C}$ for $1.5 \mathrm{~h}$. then slowly warmed to room temperature and stirred for $18 \mathrm{~h}$. The mixture was quenched with a solution of conc. $\mathrm{NH}_{4} \mathrm{OH}: \mathrm{NH}_{4} \mathrm{Cl}(1: 10,80 \mathrm{~mL})$ and stirred for $1 \mathrm{~h}$ then filtered through Celite ${ }^{\circledR}$. The filtrate was concentrated under reduced pressure, then extracted with ether $(3 \times 80 \mathrm{~mL})$. The combined ether extracts were washed with $1 \mathrm{~N} \mathrm{HCl}$, sat. $\mathrm{NaHCO}_{3}$, brine, dried $\left(\mathrm{MgSO}_{4}\right)$ and concentrated in vacuo. The crude material was purified by flash column chromatography (5:1, hexane:EtOAc) to give a colourless solid (470 mg, $73 \%$ ). A sample for microanalysis and X-ray crystallographic analysis was obtained by recrystallisation (DCM, petroleum ether) as colourless needles mp $127^{\circ} \mathrm{C} ;[\alpha]_{\mathrm{D}}+34.2\left(\mathrm{c} 1.0, \mathrm{CHCl}_{3}\right) ; v_{\max }\left(\mathrm{CHCl}_{3}\right) 1733,1716,1456,1143,1049 \mathrm{~cm}^{-1} ; \delta_{\mathrm{H}}$ (250 MHz, CDCl 3 ): 7.28 (1H, dd, J = 0.8, $1.8 \mathrm{~Hz}, \mathrm{Fu}-5 \mathrm{H}), 6.25(1 \mathrm{H}, \mathrm{dd}, \mathrm{J}=1.8,3.3 \mathrm{~Hz}, \mathrm{Fu}-4 \mathrm{H})$, $6.14(1 \mathrm{H}, \mathrm{d}, \mathrm{J}=3.3 \mathrm{~Hz}, \mathrm{Fu}-3 \mathrm{H}), 4.95-4.91(1 \mathrm{H}, \mathrm{m}, \mathrm{CHOCO}), 4.00-3.91(1 \mathrm{H}, \mathrm{m}, \mathrm{CH}-\mathrm{Fu}), 3.28$ $\left(1 \mathrm{H}, \mathrm{d}, \mathrm{J}=9.3 \mathrm{~Hz}, \mathrm{COCHCO}_{2}\right), 3.27\left(1 \mathrm{H}, \mathrm{d}, \mathrm{J}=13.3, \mathrm{HCHSO}_{2}\right), 3.30-3.11\left(2 \mathrm{H}, \mathrm{m}, 2 \mathrm{CH}-\mathrm{NSO}_{2}\right)$, $2.63\left(1 \mathrm{H}, \mathrm{d}, \mathrm{J}=13.3 \mathrm{~Hz}, \mathrm{CHSO}_{2}\right), 2.51-2.36\left(3 \mathrm{H}, \mathrm{m}, \mathrm{HCH}-\mathrm{CH}_{2} \mathrm{CO}\right), 2.01-1.56$ and 1.54-0.77 $\left(28 \mathrm{H}, \mathrm{m}, 2\left(\mathrm{CH}_{2}\right)_{5} \mathrm{CHN}, \mathrm{CHCH}_{2} \mathrm{CO}, \mathrm{CH}_{2} \mathrm{CH} 2 \mathrm{CHCH}_{2} \mathrm{CHO}\right), 1.02\left(3 \mathrm{H}, \mathrm{s}, \mathrm{CH}_{3}\right), 0.86(3 \mathrm{H}, \mathrm{s}$, $\left.\mathrm{CH}_{3}\right)$; enol: $10.83(1 \mathrm{H}, \mathrm{br}, \mathrm{s}, \mathrm{OH}), 7.21((1 \mathrm{H}, \mathrm{dd}, \mathrm{J}=0.8,1.9 \mathrm{~Hz}, \mathrm{Fu}-5 \mathrm{H}), 6.19(1 \mathrm{H}, \mathrm{dd}, \mathrm{J}=1.9$, $3.2 \mathrm{~Hz}, \mathrm{Fu}-4 \mathrm{H}), 5.85$ (1H, d, J = $3.2 \mathrm{~Hz}, \mathrm{Fu}-3 \mathrm{H}), 5.08-5.04$ (1H, m, CHOCO), 4.07-4.04 (1H, m, $\mathrm{CH}-\mathrm{Fu}), 0.76$ (3H, s, $\left.\mathrm{CH}_{3}\right), 0.44$ (3H, s, $\left.\mathrm{CH}_{3}\right) ; \delta_{\mathrm{C}}\left(250 \mathrm{MHz}, \mathrm{CDCl}_{3}\right)$ : 209.8, 166.0, 154.8, 141.4, 110.1, 105.2, 80.0, 59.3, 57.2, 53.6, 49.2, 49.1, 44.2, 39.4, 38.1, 37.2, 32.5, 30.1, 26.8, 26.3, 26.2, 26.1, 25.8, 24.9, 20.3, 19.6; enol: 177.8, 168.5, 158.4, 141.5, 103.8, 101.2, 53.64, 49.4, 48.9, 39.3, 32.73, 27.0, 20.3, 19.1; $\mathrm{C}_{32} \mathrm{H}_{47} \mathrm{O}_{6} \mathrm{NS}$ : calc C 66.99, H 8.26, N 2.44 S.5.59 Found : C 66.88, H 8.29, N 2.42, S 5.41

Methyl ester 11. A solution of 10 (80 mg, $0.14 \mathrm{mmol})$ in dry $\mathrm{MeOH}(3 \mathrm{~mL})$ was added to a Carius tube, the tube sealed under argon and heated in an oil bath at $100^{\circ} \mathrm{C}$ for $18 \mathrm{~h}$. Then the solution was cooled to room temperature and concentrated in vacuo. The residue was purified by flash column chromatography (5:1, hexane: EtOAc) to give the corresponding methyl ester $\mathbf{1 1}$ as a colourless solid (26.5 mg, $91 \%$ ). A sample for microanalysis and X-ray crystallographic analysis was obtained by recrystallisation (DCM, petroleum ether) as colourless needles mp. $46^{\circ} \mathrm{C},[\alpha]_{\mathrm{D}}+2.7\left(\mathrm{c} 0.75, \mathrm{CHCl}_{3}\right) ; v_{\max }\left(\mathrm{CHCl}_{3}\right) 1759,1728,1273,1010 \mathrm{~cm}^{-1} ; \delta_{\mathrm{H}}(250 \mathrm{MHz}$, $\left.\mathrm{CDCl}_{3}\right): 7.35$ (1H, d, J = 1.8 Hz,Fu-5H), $6.32(1 \mathrm{H}, \mathrm{dd}, \mathrm{J}=3.3,1.8 \mathrm{~Hz}, \mathrm{Fu}-4 H), 6.13(1 \mathrm{H}, \mathrm{d}, \mathrm{J}=$ $1.8 \mathrm{~Hz}, \mathrm{Fu}-3 \mathrm{H}$ ), 3.90 (1H, ddd, J = 5.8, 11.0, $10.9 \mathrm{~Hz}, \mathrm{CH}-\mathrm{Fu}), 3.77$ (3H, s, $\left.\mathrm{OCH}_{3}\right), 3.40(1 \mathrm{H}, \mathrm{d}$, $\left.\mathrm{J}=11.0 \mathrm{~Hz}, \mathrm{COCHCO}_{2} \mathrm{CH}_{3}\right), 2.59-2.43\left(3 \mathrm{H}, \mathrm{m}, \mathrm{COCH}_{2} \mathrm{HCH}\right), 2.11-2.03\left(1 \mathrm{H}, \mathrm{m}, \mathrm{COCH}_{2} \mathrm{HCH}\right)$; 
$\delta\left(250 \mathrm{MHz}, \mathrm{CDCl}_{3}\right):$ 210.0, 168.4, 154.4, 141.9, 110.2, 105.4, 60.1, 52.6, 39.5, 38.0, 26.3; Anal. Calcd for $\mathrm{C}_{11} \mathrm{H}_{12} \mathrm{O}_{4}$ : C 63.45, H 5.81 Found : C 63.42, H 5.84

Allylated ketoester 12. $\mathrm{KN}\left[\mathrm{Si}(\mathrm{Me})_{3}\right]_{2}(0.5 \mathrm{M}$ in toluene, $0.88 \mathrm{~mL}, 0.44 \mathrm{mmol})$ was added dropwise to a solution of methyl ester $11(68 \mathrm{mg}, 0.32 \mathrm{mmol})$ in dry THF $(6 \mathrm{~mL})$ under nitrogen at $-78^{\circ} \mathrm{C}$. The solution was stirred for $1 \mathrm{~h}$ then allyl bromide $(0.4 \mathrm{~mL}, 4.61 \mathrm{mmol})$ was added. The reaction was stirred at $-78^{\circ} \mathrm{C}$ for $2 \mathrm{~h}$, then slowly warmed to room temperature and stirred for $12 \mathrm{~h}$. The mixture was quenched with sat. $\mathrm{NH}_{4} \mathrm{Cl}(5 \mathrm{~mL})$ and extracted with ether $(3 \times 5 \mathrm{~mL})$. The combined ether extracts were washed with saturated $\mathrm{NaHCO}_{3}$, brine and dried $\left(\mathrm{MgSO}_{4}\right)$ then concentrated under reduced pressure. The residue was purified by column chromatography (4:1 light petroleum ether) to afford a colourless solid (71 mg, 89\%). A sample for microanalysis and X-ray crystallographic analysis was obtained by recrystallisation (DCM, petroleum ether) as colourless needles mp $46^{\circ} \mathrm{C}$, $[\alpha]_{\mathrm{D}}-99.4$ (c 1.3, $\mathrm{CHCl}_{3}$ ); $v_{\max }(\mathrm{DCM})$ 1752, 1736, 1231, 1179 $\mathrm{cm}^{-1} ; \delta_{\mathrm{H}}\left(250 \mathrm{MHz}, \mathrm{CDCl}_{3}\right): 7.32(1 \mathrm{H}, \mathrm{d}, \mathrm{J}=1.9 \mathrm{~Hz}, \mathrm{Fu}-5 H), 6.29(1 \mathrm{H}, \mathrm{dd}, \mathrm{J}=1.9,3.2 \mathrm{~Hz}, \mathrm{Fu}-$ $4 H$ ), 6.09 (1H, d, J = 3.2 Hz, Fu-3프), 5.69-5.53 (1H, m, $\mathrm{CH}=\mathrm{CH} 2)$, 5.28-5.17 (2H, m, $\mathrm{CH}=\mathrm{CH}_{2}$ ), $3.62(1 \mathrm{H}, \mathrm{dd}, \mathrm{J}=6.0,12.0 \mathrm{~Hz}, \mathrm{CH}-\mathrm{Fu}), 3.52\left(3 \mathrm{H}, \mathrm{s}, \mathrm{OCH}_{3}\right), 2.80-2.42\left(3 \mathrm{H}, \mathrm{m}, \mathrm{COCH}_{2} \mathrm{HCHFu}\right.$ ), 2.27-2.15 (1H, m, HCHCHFu); $\delta_{\mathrm{C}}\left(250 \mathrm{MHz}, \mathrm{CDCl}_{3}\right): 214.1,170.1,153.2,142.1,132.6,120.7$, 110.1, 106.3, 63.4, 52.1, 42.2, 38.8, 35.5, 24.1; Anal. Calcd for $\mathrm{C}_{14} \mathrm{H}_{16} \mathrm{O}_{4^{-}}$; C 63.45, H 5.81 Found : C 63.42, H 5.84

(2S, 3R) 2-allyl-3-(2-furyl)cyclopentanone (13). Lithium chloride (30 $\mathrm{mg}, 0.70 \mathrm{mmol}$ ) was added to a solution of $12(50 \mathrm{mg}, 0.20 \mathrm{mmol})$ in dry HMPA (3 mL) under nitrogen. The solution was heated to $80^{\circ} \mathrm{C}$ for $12 \mathrm{~h}$. After cooling to room temperature, $2 \mathrm{M} \mathrm{HCl} \mathrm{(5} \mathrm{mL)} \mathrm{was} \mathrm{added} \mathrm{and}$ the mixture extracted with ether $(3 \times 5 \mathrm{~mL})$. The combined ether extracts were washed with $1 \mathrm{M}$ $\mathrm{HCl}$, brine, dried $\left(\mathrm{MgSO}_{4}\right)$ and concentrated in vacuo. The crude product was purified by flash column chromatography (4:1, light petroleum ether, ether) to give pure trans isomer $\mathbf{1 3}$ as a colourless oil (28 mg, 74\%) $[\alpha]_{\mathrm{D}}-55.5$ (c 1.0, $\left.\mathrm{CHCl}_{3}\right) ; \delta_{\mathrm{H}}\left(400 \mathrm{MHz}, \mathrm{CDCl}_{3}\right): 7.36(1 \mathrm{H}, \mathrm{dd}, \mathrm{J}=$ 0.8, 1.9 Hz, Fu-5H), 6.33 (1H, dd, J = 1.9, 3.2 Hz, Fu-4H), 6.09 (1H, d, J = 3.2 Hz, Fu-3H), 5.68$5.56\left(1 \mathrm{H}, \mathrm{m}, \mathrm{CH}=\mathrm{CH}_{2}\right), 5.08-5.00\left(2 \mathrm{H}, \mathrm{m}, \mathrm{CH}=\mathrm{CH}_{2}\right), 3.21(1 \mathrm{H}, \mathrm{dt}, \mathrm{J}=6.4,10.7 \mathrm{~Hz}, \mathrm{CH}-\mathrm{Fu})$, 2.53-2.27 (5H,m), 2.24-2.14 (1H, m), 2.09-1.98 (1H, m); $\delta_{\mathrm{C}}\left(250 \mathrm{MHz}, \mathrm{CDCl}_{3}\right): \delta 218.1,156.0$, 141.4, 134.5, 117.5, 110.1, 105.1, 53.5, 39.8, 37.9, 31.9, 26.6; HRMS Found 191.1078; $\mathrm{C}_{12} \mathrm{H}_{15} \mathrm{O}_{2}^{+}\left(\mathrm{MH}^{+}\right)$requires 191.1072

\section{Acknowledgments}

V. C. thanks the Royal Thai Government for a studentship. . We thank EPSRC and the University of Reading for funds for the Image Plate system. 


\section{References and Notes}

1. Evans, F. J.; Soper, C. J. Lloydia 1978, 41, 193.

2. (a) Ohigashi, H.; Kawazu, K.; Koshimizu, K.; Mitsui, T. Agr. Biol. Chem. 1972, 36, 2529.

(b) Kelly, W. R.; Bick, I. R. C. Res. Vet. Sci. 1976, 20, 311. (c) Evans, F. J. Naturally Occurring Phorbol Ester, CRC. Press, 1986.

3. (a) Nishizuka, Y. Nature 1984, 308, 693. (b) Rosfjord, E. C.; Maemura, M.; Johnson, M. D.; Torri, J. A.; Akiyama, S. K. Woods, Jr. V. L.; Dickson, R. B. Exp. Cell. Res. 1999, 248, 260.

4. (a) Wender, P. A.; Mascareñas, J. L. J. Org. Chem. 1991, 56, 6267. (b) Wender, P. A.; Kogen, H.; Lee, H. Y.; Junger, J. D.; Wilheim, R.S.; Williams, P. D. J. Am Chem. Soc. 1989, 111, 8957. (c) Wender, P. A.; Kogen, H.; Lee, H. Y.; Junger, J. D. Wilheim, R.S.; Williams, P. D. J. Am Chem. Soc. 1989, 111, 8954. (d) Wender, P. A.; Keenan, R. M.; Lee, H. Y. J. Am Chem. Soc. 1987, 109, 4390;

5. (a) Page, P. C. B.; Jennens, D. C.; Porter, R. A.; Baldock, A. N. Synlett 1991, 472. (b) Page, P. C. B.; Jennens, D. C. J. Chem. Soc., Perkin Trans. I 1992, 2587. (c) Page, P. C. B.; Jennes, D. C.; McFurland, H. Tetrahedron Lett. 1997, 38, 5395. (d) Rigby J. H.; Kierkus, P. Ch. J. Am. Chem. Soc. 1989, 111, 4125. (e) Rigby, J. H.; Kierkus, P. Ch.; Head, D. Tetrahedron Lett. 1989, 30, 5073. (f) Dauben, W. G.; Dinges, J.; Smith, T. C. J. Org. Chem. 1993, 58, 7635. (g) Mcmills, M. C.; Zhuang, L.; Wright, D. L; Watt, W. Tetrahedron Lett. 1994, 35, 8311. (h) Paquett, L. A.; Sauer, D. R.; Edmondson, S. D.; Friedrich, D. Tetrahedron 1994, 50, 4071. (i) McLoughlin, J. I.; Brahma, R.; Campopiano, O.; Little, R. D. Tetrahedron Lett. 1990, 31, 1377.

6. (a) Wender, P. A.; Rice K. D.; Schnute, M. E. J. Am Chem. Soc. 1997, 119, 7897. (b) Shigeno, K.; Ohne, K.; Yamaguchi, T.; Sasai, H.; Shibasaki, M. Heterocycles 1992, 33, 161. (c) Shigeno, K.; Sasai, H.; Shibasaki, M. Tetrahedron Lett. 1992, 32, 4937. (d) Sugita, K.; Shigeno, K.; Neville, C. F.; Sasai, H.; Shibasaki, M. Synlett 1994, 325. (e) Tokunoh, R.; Tomiyama, H.; Sodeoka, M.; Shibasaki, M. Tetrahedron Lett. 1996, 37, 2449.

7. (a) Harwood, L. M.; Brickwood, A. C.; Morisson, V.; Robertson, J.; Swallow, S. J. Heterocyclic Chem. 1999, 36, 1391. (b) Brickwood, A. C.; Drew, M. G. B.; Harwood, L. M.; Ishikawa, T.; Marais, P.; Morisson, V. J. Chem. Soc., Perkin Trans. 1. 1999, 913. (c) Harwood, L. M.; Ishikawa, T.; Phillips, H.; Watkin, D. J. Chem. Soc., Chem Commun 1991, 527. (d) Harwood, L. M.; Jackson, B.; Jones, G.; Prout, K.; Thomas, R. M.; Witt, F. J. J. Chem. Soc., Chem. Commun. 1990, 608. (e) Harwood, L. M.; Jones, G.; Pickard, J.; Thomas R. M.; Watkin, D. J. Chem. Soc., Chem. Commun. 1990, 605.

8. Oppolzer, W.; Dudfield, P.; Stevenson, T.; Godel, T. Helv. Chim. Acta. 1985, 68, 212.

9. Kantam, M. L.; Neeraja, V.; Bharathi, B.; Reddy, Ch. V. Catalysis Letters 1999, 62, 67.

10. Urban, E.; Knühl, G.; Helmchen, G. Tetrahedron 1996, 52, 971.

11. Ng, J. S.; Behling, J. R.; Campbell, A. L. Tetrahedron. Lett. 1988, 29, 3045.

12. Data for all 3 crystals were collected with $\mathrm{MoK}_{\alpha}$ radiation using the MAResearch Image Plate System. The crystals were positioned at $70 \mathrm{~mm}$ from the Image Plate. 95 frames were 
measured at $2^{\circ}$ intervals with a counting time of 2 minutes. Data analysis was carried out with XDS program. ${ }^{12 a}$ The structures were determined by direct methods using the SHELX86 program. ${ }^{12 b}$ The non-hydrogen atoms were refined with anisotropic thermal parameters. The hydrogen atoms bonded to carbon were included in geometric positions and given thermal parameters equivalent to 1.2 times those of the atom to which they were attached. The structures were then refined by full-matrix least-squares on $F^{2}$ using the SHELXL program. ${ }^{12 c}$ Data can be obtained at www.ccdc.cam.ac.uk/conts/retrieving.html [or from the Cambridge Crystallographic Data Centre, 12, Union Road, Cambridge CB2 1EZ, UK; fax: (internat.) +44-1223/336-033; E-mail: deposit@ccdc.cam.ac.uk. Reference numbers are CCDC 192821 for 10, CCDC 192822 for 11 and CCDC 192823 for 12. (a) W. Kabsch, J. Appl. Crystallogr. 1988, 21, 916. (b) Sheldrick, G. M. Acta Cryst., Sect. A 1990, 46, 467. (c) G. M. Sheldrick, SHELXL, Program for Crystal Structure Refinement, University Göttingen, 1993.

13. Hashimoto, S.; Watanabe, N.; Kawano, K.; Ikegami, S. Synth. Comm. 1994, 24, 3277.

14. Urban, E.; Knühl G.; Helmchen, G. Tetrahedron 1995 51, 13031.

15. Muller, P.; Siegfried, B. Tetrahedron Lett. 1973, 37, 3565. 